\title{
Fixação esquelética externa em fratura tarsometatársica de seriema (Cariama cristata): relato de caso
}

\author{
External skeletal fixation in tarsumetatarsus fracture of red-legged \\ seriema (Cariama cristata): a case report \\ B.B.J. Torres ${ }^{1}$, L.A.L. Muzzi $^{2}$, E.G.L. Alves ${ }^{1}$, C.A.L. Leite $^{2}$, M.A.R. Feliciano ${ }^{2}$, \\ G.R. Sampaio ${ }^{2}$, R.A.L. Muzzi ${ }^{2}$. \\ ${ }^{1}$ Aluno de pós-graduação - EV-UFMG - Belo Horizonte, MG \\ ${ }^{2}$ Universidade Federal de Lavras - UFLA
}

\begin{abstract}
RESUMO
Uma seriema (Cariama cristata) adulta foi atendida com histórico de traumatismo por tentativa de captura. A ave apresentava dificuldade de apoio do membro pélvico direito, dor à manipulação e fratura exposta do osso tarsometatarso. Optou-se pelo tratamento cirúrgico com redução fechada, utilizando-se fixador esquelético externo tipo II, com barra de conexão acrílica. A técnica cirúrgica utilizada foi satisfatória para o tratamento da fratura, possibilitando reparação óssea e retorno funcional do membro 60 dias após a cirurgia.
\end{abstract}

Palavras-chave: seriema, fixação esquelética externa, fratura exposta, tarsometatarso

\begin{abstract}
An adult red-legged seriema (Cariama cristata) was referred for examination with history of trauma by capture. The physical examination revealed lameness in the right pelvic limb, sensibility to touch and open fracture of tarsumetatarsus. The treatment was done with surgical closed reduction using a external skeletal fixator type II with acrylic connecting bar. The surgical technique applied was satisfactory for the treatment of the fracture of tarsumetatarsus, since there was bone healing and functional return of the limb at 60 days after surgery.
\end{abstract}

Keywords: red-legged seriema, external skeletal fixation, open fracture, tarsumetatarsus

\section{INTRODUÇÃO}

Entre as várias doenças que podem acometer as aves, as fraturas de ossos longos das asas e dos membros pélvicos estão entre as mais freqüentes (Mccartney, 1994; Harcour-Brown, 2002). Os objetivos do reparo das fraturas aviárias são semelhantes aos dos mamíferos, incluindo alinhamento e aposição dos fragmentos ósseos, manutenção da biomecânica da estrutura afetada, obtenção de rígida estabilização após redução, e retorno precoce à função (Westfall e Egger, 1979; Maccoy, 1991; Alievi et al., 1998; Freitas et al., 2003).

Diversos métodos têm sido utilizados para o tratamento das fraturas em aves, entretanto, devido às variações no tamanho, peso e conformação da ave, à anatomia óssea peculiar, à alta incidência de fraturas complicadas e às diferenças das necessidades funcionais entre espécies e indivíduos, não é possível estabelecer

Recebido em 16 de agosto de 2005

Aceito em 23 de novembro de 2006

E-mail: brunobjtorres@yahoo.com.br

Endereço para correspondência (corresponding address)

Rua Ararirama, 97 - 31310-330 - Belo Horizonte, MG 
ainda um método ideal de osteossíntese para os ossos longos desses animais (Alievi et al., 2002; Freitas et al., 2003; Castro et al., 2004).

O uso de bandagens e talas, aparelhos de fixação esquelética externa, associação de pinos intramedulares e cerclagens, e utilização de placas e parafusos são algumas das opções disponíveis para a osteossíntese em aves (Maccoy, 1992; Alievi et al., 1998; Freitas et al., 2003; Teixeira et al., 2004). Convém ressaltar que o uso prolongado da imobilização externa em aves pode levar à "doença da fratura" que se caracteriza por atrofia muscular, rigidez articular, osteoporose e não-união óssea, sendo essa uma das principais causas de fracasso na ortopedia aviária (Alievi et al., 2001; Leotte et al., 2004).

Os fixadores esqueléticos externos são indicados em casos de fraturas expostas com lesões dos tecidos moles (Freitas et al., 2003). Um dos benefícios de sua utilização é a possibilidade de aplicação da técnica de redução óssea fechada, mantendo o suprimento sanguíneo no local da fratura e reduzindo o tempo cirúrgico (Kuzma, 1992; Dudley et al., 1997).

O aparelho de fixação esquelética externa deve permitir o uso controlado do membro fraturado durante o período de convalescença, pois pequenos movimentos nessa fase minimizam a atrofia por desuso e promovem maior precocidade ao retorno funcional do membro (Levitt, 1989; Gaiga e Schossler, 2003).

O trabalho teve o objetivo de descrever o tratamento cirúrgico de um caso de fratura exposta do osso tarsometatarso direito em seriema, utilizando-se redução fechada da fratura e imobilização óssea com fixador esquelético externo tipo II com barra de conexão acrílica.

\section{CASUÍSTICA}

Uma seriema adulta, de $74 \mathrm{~cm}$ de altura, pesando $1,7 \mathrm{~kg}$, foi atendida com histórico de traumatismo por tentativa de captura. Ao exame clínico, a ave apresentava dificuldade de apoio do membro pélvico direito, dor à manipulação, fratura exposta do osso tarsometatarso e lesão dos tecidos moles adjacentes. $\mathrm{O}$ animal foi submetido a tranqüilização com diazepam na dose de $0,2 \mathrm{mg} / \mathrm{kg}$, pela via intramuscular (músculo peitoral), para realização dos exames radiográficos convencionais do membro acometido, nas projeções craniocaudal e mediolateral. À avaliação radiográfica, observouse uma fratura simples, completa, transversa no terço distal do osso tarsometatarso do membro direito, com desvio discreto do eixo ósseo.

Optou-se pela redução e imobilização cirúrgica da fratura. A indução anestésica foi feita com a associação de xilazina e quetamina, nas doses de $3 \mathrm{mg} / \mathrm{kg}$ e $15 \mathrm{mg} / \mathrm{kg}$, respectivamente, via intramuscular. A anestesia foi mantida com aplicações subseqüentes da mesma associação de anestésicos, utilizando-se metade da dose inicial de indução. A ave foi posicionada em decúbito lateral esquerdo e procedeu-se à anti-sepsia do campo cirúrgico. Foi realizada a redução fechada do foco de fratura, seguida pela imobilização óssea utilizando-se fixação esquelética externa (Fig. 1). Com o auxílio de uma furadeira de baixa rotação e sob irrigação constante, foram introduzidos transcorticalmente quatro pinos de Steinmann com diâmetro de $2 \mathrm{~mm}$, dois proximais e dois distais ao foco da fratura, no sentido de lateral para medial. Os pinos mais distantes do foco da fratura foram introduzidos paralelamente às superfícies articulares adjacentes, enquanto os pinos mais próximos ao foco foram introduzidos com angulação de aproximadamente $70^{\circ}$ em relação ao eixo longitudinal do osso (Fig. 2). Posteriormente, alinhou-se o eixo ósseo e os pinos foram fixados externamente com emprego de barras acrílicas de metilmetacrilato, posicionadas nas faces medial e lateral do membro (Fig. 1). É importante ressaltar que antes da colocação das barras acrílicas, as extremidades dos pinos foram dobradas com auxílio de dois alicates cirúrgicos, um apoiando o pino para não desestabilizá-lo, e o outro utilizado para dobrar o pino. Imediatamente ao término do procedimento cirúrgico, radiografou-se o membro operado em incidência craniocaudal (Fig. 2). 


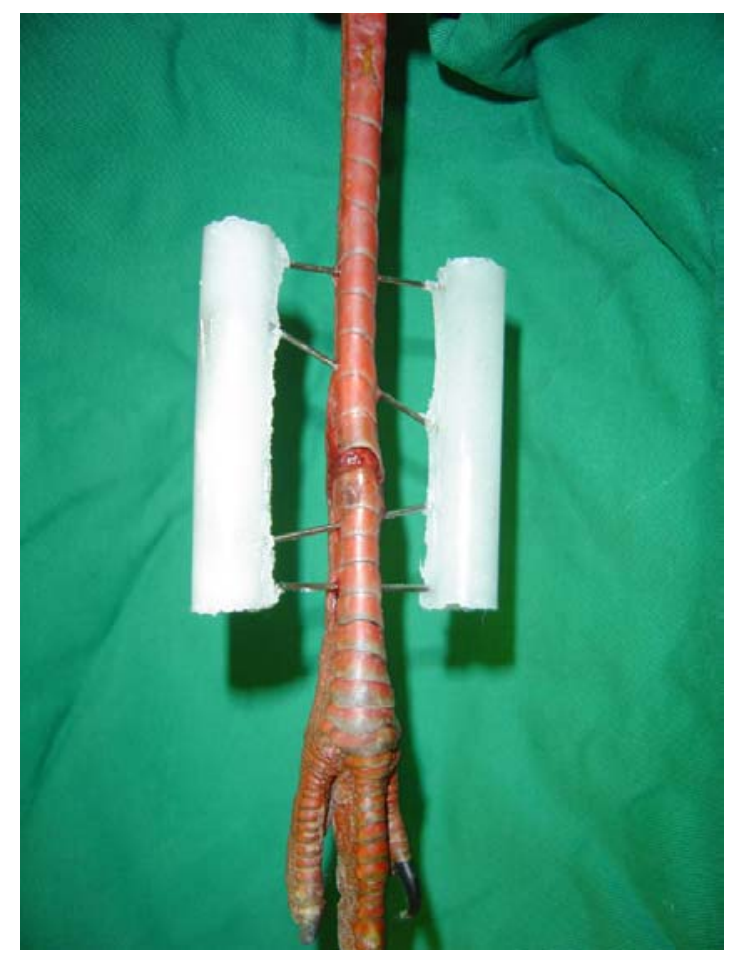

Figura 1. Osteossíntese de tarsometatarso direito de seriema. Utilizou-se redução fechada com fixador esquelético externo tipo II. Após o alinhamento do eixo ósseo, os pinos foram fixados externamente com emprego de barras acrílicas de metilmetacrilato, posicionadas nas faces medial e lateral do membro.

No período pós-operatório, a ave foi mantida em viveiro de $7,5 \mathrm{~m}^{3}$ e alimentada com camundongos de biotério, ovos, ração para galinhas poedeiras e pintos de um dia. Foram administrados flunixina meglumina na dose de $2 \mathrm{mg} / \mathrm{kg}$, por via intramuscular, a cada 24 horas, por sete dias consecutivos, e penicilina benzatina na dose de 50.000UI, por via intramuscular, a cada 48 horas, por três aplicações. Diariamente, foi realizada limpeza em torno dos pinos com solução fisiológica e iodopovidine, e aplicada bandagem protetora. A seriema foi avaliada semanalmente por exames clínicos específicos. A reparação da fratura foi acompanhada por meio de radiografias quinzenais, obtidas nas projeções craniocaudal e mediolateral, até 60 dias após a cirurgia.

\section{DISCUSSÃO}

O protocolo anestésico empregado, que preconizou a utilização da anestesia dissociativa

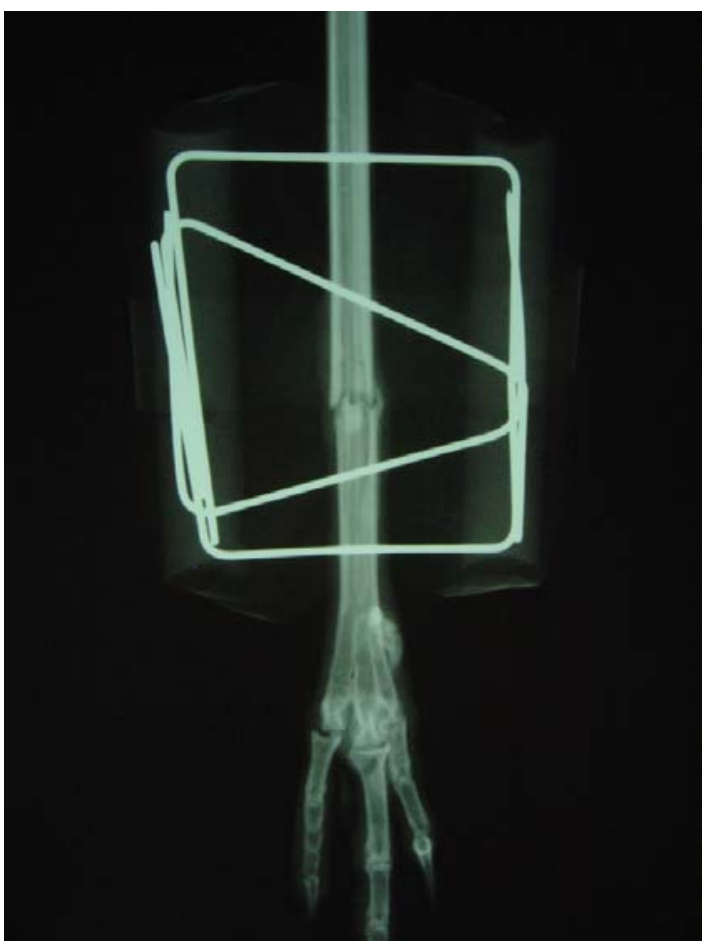

Figura 2. Radiografia craniocaudal de tarsometatarso direito de seriema no pós-operatório imediato, mostrando fixador esquelético externo tipo II. Os pinos mais distantes do foco da fratura foram introduzidos paralelamente às superfícies articulares adjacentes, enquanto os pinos mais próximos ao foco foram introduzidos com angulação de aproximadamente $70^{\circ} \mathrm{em}$ relação ao eixo longitudinal do osso.

com fármacos aplicados pela via intramuscular, demonstrou ser uma opção viável e prática para a indução e manutenção da anestesia em cirurgia ortopédica de seriema, embora Guimarães e Moraes (2000) tenham recomendado a manutenção anestésica de aves por meio de agentes inalatórios.

Os quatro pinos de Steinmann, com diâmetro de $2 \mathrm{~mm}$, foram suficientes para manter a estabilidade do foco da fratura. Segundo Alievi et al. (2001), para a confecção dos aparelhos de fixação esquelética externa, o diâmetro dos pinos deve ser entre 20 e $30 \%$ do diâmetro ósseo. Outro aspecto importante na implantação do aparelho de fixação é a angulação dos pinos. Quando todos os pinos são implantados num ângulo reto em relação ao eixo longitudinal do osso, aumenta a possibilidade de afrouxamento desses, levando à falha do método (Alievi et al., 2001). No presente relato, os dois pinos mais próximos ao foco da fratura foram inseridos 
numa angulação de aproximadamente $70^{\circ} \mathrm{em}$ relação ao eixo ósseo, possibilitando que o aparelho de fixação óssea permanecesse estável até 45 dias após a cirurgia.

O tipo de fratura e o discreto desvio do eixo ósseo do tarsometatarso permitiram que fosse realizada a redução fechada do foco da fratura. Essa manobra cirúrgica apresenta vantagens em relação à redução aberta, já que impede o rompimento do hematoma da fratura, mantém o suprimento sangüíneo e diminui os riscos de osteomielite. Além disso, segundo Kuzma (1992) e Dudley et al. (1997), a redução fechada resulta em menor tempo cirúrgico, pois elimina o tempo necessário para exposição e reconstrução dos fragmentos ósseos, diminuindo o período anestésico e os riscos de morte para pacientes gravemente traumatizados. Ademais, o simples fato de a fratura estar exposta facilitou a sua redução, sendo, por mais esse motivo, desnecessária a abertura do foco.

Aos 15 dias após a intervenção cirúrgica, a ave já apresentava uso parcial do membro, com apoio de peso sobre o membro operado, embora radiograficamente ainda não fosse possível a observação de calo ósseo. Clinicamente, observa-se que a reparação das fraturas em aves ocorre mais rapidamente do que em mamíferos (Teixeira et al., 2004). Wissman (1999) relatou que os sinais radiográficos de reparação óssea em aves ocorrem mais tardiamente do que os sinais clínicos de consolidação da fratura, devido à rápida formação de calo constituído por cartilagem hialina, o que proporciona união e estabilidade aos fragmentos ósseos, mesmo mantendo a linha de fratura radiograficamente visível. O calo endosteal é o que mais contribui para a reparação das fraturas em aves, e implantes de metal colocados ao redor do local de fratura podem enfraquecer a formação desse tipo de calo ósseo (Bush, 1986). O método de fixação esquelética externa utilizado neste caso parece não ter interferido na formação do calo ósseo endosteal.

Aos 30 dias, já podia ser visibilizado o início da formação de calo ósseo. Aos 45 dias, havia presença de calo ósseo exuberante, entretanto, notava-se reação periosteal intensa e áreas de lise óssea no trajeto dos pinos. Nesta fase, o foco da fratura apresentava-se estável, e o aparelho de fixação esquelética demonstrava mobilidade à manipulação, principalmente nos pinos proximais, sendo removido o aparelho fixador. A lise óssea ao redor dos pinos é a complicação mais freqüente quando se utilizam os fixadores esqueléticos externos (Egger, 1991), sendo radiograficamente visível a partir do $30^{\circ}$ dia da colocação do aparelho (Johnson et al., 1989). O pino proximal é o mais comumente afetado por tal problema, por ser sobre esse pino exercida a maior força quando o animal apóia o membro, resultando, com isso, em necrose óssea no local de inserção do pino (Gumbs et al., 1988). Segundo Johnson et al. (1989), Egger (1991) e Lewis e Bloomberg (1994), a drenagem no local de inserção do pino e o seu afrouxamento, além da reação periosteal, são complicações comuns com o uso dessa técnica de osteossíntese. Normalmente esses problemas estão relacionados entre si e produzem incômodo ao animal, diminuindo, com isso, o uso do membro operado (Lewis e Bloomberg, 1994).

Após a retirada dos pinos, pôde-se notar que o calo ósseo presente já proporcionava boa estabilidade ao foco da fratura, contudo, uma tala de coaptação foi colocada e mantida por 15 dias, devido à possível fragilidade óssea nos locais dos trajetos dos pinos. Aos 60 dias de pós-operatório (Fig. 3), foi realizada nova avaliação radiográfica, que mostrou calo ósseo em processo de organização, atenuação da linha de fratura e considerável reação periosteal, com diminuição da lise óssea no trajeto dos pinos. Segundo Williams et al. (1987), que observaram a reparação óssea de fraturas experimentais em pombos, foram encontradas evidências de união periosteal após três semanas e completa união óssea na sexta semana após a fixação esquelética.

Confirmando os resultados de Bush (1977) e Levitt (1989), o método de fixação esquelética externa utilizado nessa ave proporcionou boa estabilidade ao foco da fratura, permitindo adequada reparação óssea, evidenciada pelos exames físicos e radiográficos. Além disso, o método de fixação empregado permitiu a utilização precoce do membro afetado, o que minimizou a atrofia por desuso. 


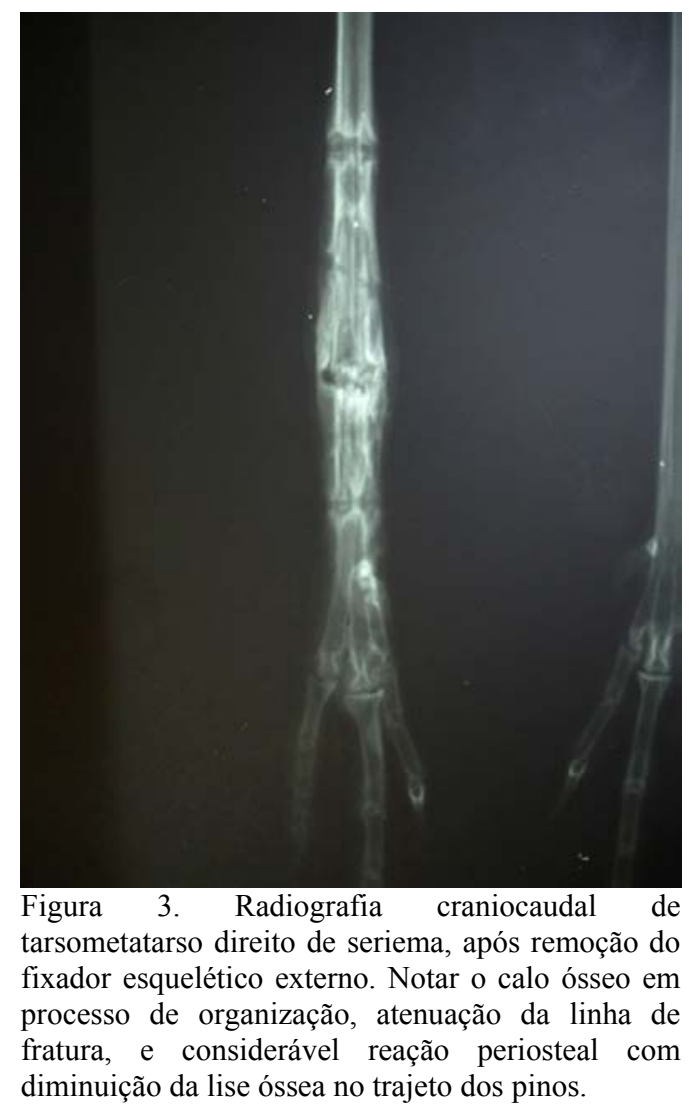

\section{REFERÊNCIAS BIBLIOGRÁFICAS}

ALIEVI, M.M.; SCHOSSLER, J.E.; TEIXEIRA, M. Osteossíntese de úmero em arara-canindé: relato de caso. Clin. Vet., n.15, p.18-20, 1998.

ALIEVI, M.M.; SCHOSSLER, J.E.; HIPPLER, R.A. et al. Redução fechada e fixação esquelética externa tipo I para tratamento de fraturas de tibiotarso em pombos domésticos (Columba livia). Arq. Bras. Med. Vet. Zootec., v.54, p.259266, 2002.

ALIEVI, M.M.; SCHOSSLER, J.E.; HIPPLER, R.A. et al. Redução fechada e fixação esquelética externa tipo II para o tratamento de fraturas de tibiotarso em pombos domésticos (Columba livia) Cienc. Rural, v.31, p.1019-1025, 2001.

BUSH, M. Laparoscopy and surgery. In: FOWLER, M.E. Zoo \& Wild Animal Medicine. 2.ed. Philadelphia: W.B. Saunders, 1986. p.254-261.

BUSH, M. External fixation of avian fractures. J. Am. Anim. Hosp. Assoc., v.171, p.943-946, 1977.

CASTRO, P.F.; MATERA, J.M.; FANTONI, D.T. et al. Uso de pino de aço intramedular na reparação de fraturas de ossos longos em psitacídeos: arara-azul (Anodorhynchus hyacinthinus), arara-canindé (Ara ararauna) e papagaioverdadeiro (Amazona aestiva). Clin. Vet., n.52, p.56-64, 2004.

DUDLEY, M.; JOHNSON, A.L.; OLMSTEAD, M. et al. Open reduction and bone plate stabilization compared with closed reduction and external fixation, for treatment of comminuted tibial fractures: 47 cases (1980-1995) in dogs. $J$. Am. Vet. Med. Assoc., v.211, p.1008-1012, 1997.

EGGER, E.L. Complications of external fixation. A problemoriented approach. Vet. Clin. N. Am.: Small Anim. Pract., v.21, p.705-733, 1991.

FREITAS, S.H.; PIRES, M.A.M.; CARVALHO, H.S. et al. Redução fechada e fixador externo em fratura umeral de ema (Rhea americana). Relato de caso. Clin. Vet., n.45, p.40-42, 2003.

GAIGA, L.H.; SCHOSSLER, J.E.W. Osteossíntese de úmero por xenoenxerto ósseo preservado em mel em pombos domésticos (Columba livia). Cienc. Rural, v.33, p.709-715, 2003 .

GUIMARÃES, L.D.; MORAES, A.N. Anestesia em aves: agentes anestésicos. Cienc. Rural, v.30, p.1073-81, 2000.

GUMBS, J.M.; BRINKER, W.O.; DECAMP, C.E. et al. Comparison of acute and chronic pull-out resistance of pins used with the external fixator (Kirschner splint). J. Am. Anim. Hosp. Assoc., v.24, p.231-234, 1988.

HARCOUR-BROWN, N.H. Orthopedic conditions that affect the avian pelvic limb. Vet. Clin. N. Am.: Exotic Anim. Pract., v.5, p.49-81, 2002.

JOHNSON, A.L.; KNELLER, S.K.; WEIGEL, R.M. Radial and tibial fracture repair with external skeletal fixation: effects of fracture type, reduction, and complications on healing. Vet. Surg., v.18, p.367-372, 1989.

KUZMA, A. Avian orthopedics: an overview. In: AMERICAN COLLEGE OF VETERINARY SURGEONS, 1992, Miami, Florida. Proceedings... Miami: ACVS, 1992. p.625-627.

LEOTTE, A.M.; RAISER, A.G.; BRAGA, F.A. et al. Fixação esquelética externa tipo Ia (unilateral-uniplanar) para osteossíntese diafisária de úmero em pombos domésticos (Columba livia). Cienc. Rural, v.34, p.1865-1870, 2004.

LEVITT, L. Avian orthopedics. Comp. Cont. Educ. Pract. Vet., v.11, p.899-910, 1989.

LEWIS, D.D.; BLOOMBERG, M.S. Fijación ósea externa. Waltham Focus, v.4, p.2-18, 1994.

MACCOY, D.M. General principles of avian surgery. Comp. Small Anim., v.13, p.989-992, 1991.

MACCOY, D.M. Treatment of fractures in avian species. Vet. Clin. N. Am.: Small Anim. Pract., v.22, p.225-238, 1992.

MCCARTNEY, W.T. Orthopaedics injuries in pigeons. Vet. Rec., v.134, p.305-307, 1994.

TEIXEIRA, C.R.; RAHAL, S.C.; LIMA, A.F.M. et al. Haste intramedular de polipropileno, combinada ou não a biomateriais, no tratamento de fraturas induzidas no úmero de pombos. Arch. Vet. Sci., v.9, p.67-72, 2004.

WESTFALL, M.L.; EGGER, L.E. The management of long bone fractures in birds. Iowa State Vet., v.41, p.81-87, 1979.

WILLIANS, R.; HOLLAND, M.; MILTON, J.L. et al. A comparative study of treatment methods for long bone fractures. Comp. Anim. Pract., v.1, p.48-55, 1987.

WISSMAN, M.A. New tools, diagnostics aid in bone and beak repair in birds. Vet. Prod. News, v.11, p.44-45, 1999 